\title{
Intrinsic phase coherence of laser light
}

\author{
David T. Pegg* \\ Centre for Quantum Computer Technology, \\ School of Biomolecular and Physical Sciences, \\ Griffith University, Nathan, Brisbane 4111, Australia
}

(Dated: April 15, 2009)

\begin{abstract}
We examine the phase relationships between different packets of a beam of light emitted from a cavity through a partially transmitting mirror. We find that the beam has a pronounced phase coherence even when the intra-cavity field has a completely indeterminate phase as, for example, when it is in a pure photon number state. In this case, the phase coherence can be directly attributed to entanglement caused by the partially transmitting mirror and, even though the external light has phase coherence, it has no identifiable phase. Consequently there is no phase diffusion but there is diffusion of the phase difference between a packet and the intra-cavity field. Remarkably, we find that the phase coherence of the beam is almost independent of the intra-cavity state, provided this has a reasonably narrow number state distribution about a large mean value.

PACS numbers: 42.50.-p, 42.55.Ah
\end{abstract}

${ }^{*}$ D.Pegg@griffith.edu.au 


\section{INTRODUCTION}

In recent years there has been a renewed interest in the nature of laser light. It has been commonly accepted that single-mode laser light is in a coherent state and indeed this acceptance leads to very successful calculations of experimental results. For a typical laboratory laser, that is a cavity with a fully reflecting mirror at one end and a partially transmitting mirror at the other, it is not difficult to show that, in order to emit a coherent state into the external mode, the field in the internal cavity mode must also be in a coherent state. Much of the early theoretical development involved finding the internal field. Various methods were used but in most cases the final cavity state above threshold was found by tracing over the incoherently excited atoms of the cavity. This left an improper mixture with a Poissonian photon number state distribution. The gap between this result and a pure internal coherent state can be easily bridged, at least mathematically, by expanding the density operator for the improper mixture as an integral of coherent states over all possible mean phases. This is the same mathematical expression for the density operator that one would use to describe a coherent state if one had no knowledge of, or had forgotten, the mean phase. We should note that in speaking of phase we mean relative to some separate reference system such as field whose phase is defined as zero, or perhaps relative to some form of clock. This expansion of the density operator is a special case of a more general result that any density operator that is diagonal in the number state representation, with the exception of a pure number state, can be expressed as a mixture of partial phase states each with a preferred phase [1].

While it might seem strange that an incoherently excited atomic system can give a field that is in a pure state of definite but unknown phase, this assumption does lead to an external field in a coherent state and to the successful prediction of experimental results. One of the early predictions was the interference of light from two independent lasers, which was detected by Pfleegor and Mandel [2, 3]. Interestingly, even though their experiments have been taken as evidence that laser light is in a coherent state, Pfleegor and Mandel actually cautioned against this conclusion. They advocated that it is better to associate the interference with the detection process itself and state that similar results should be obtainable with a wide range of possible states of the two fields being superposed. Further experimental and theoretical work [1,4-7] verified this. Pfleegor and Mandel showed that 
the detection of the first photon changes the combined field state in such a way that the expression for the probability of detection for the next photon contains interference terms. In effect, the detection of the first photon breaks the phase symmetry. A later related interference experiment by Ghosh and Mandel [8] has been interpreted in terms of the first photon detection event reducing the state of the two fields to a simple entangled state that has a reasonably well-defined phase difference, giving the observed interference effect [9]. Later Javanainen and Yoo [10] and Horak and Barnett [11] looked at the interference for two Fock states, but in the context of Bose-Einstein condensates, in a similar way. The issue was revived again in the optical context in an important paper by Mølmer [6] who showed that states such as Fock states lead to balanced homodyne signals that look like those of coherent states. This was part of a strong argument presented by Mølmer that optical coherences as one would find, for example, if laser light were in a coherent state, are actually just convenient fiction. This stimulated a controversial debate on the subject [12-21]. It is generally agreed that coherent states are a convenient mathematical tool for calculating results but opinions are divided as to whether or not light from a laboratory laser is actually in such a state, which would imply that it has acquired a reasonably well-defined phase relative to some separate phase reference.

It is not just Poisson number state distributions, of course, that can be expressed in terms of coherent states. For more general states the Glauber-Sudarshan P-representation can be used $[22,23]$ and pure states such as Fock states can be written as superpositions of coherent states [16]. The convenience of using coherent states for calculations arises from their simplicity. A coherent state in the intra-cavity mode will produce a coherent state outside the cavity. The main effect of the partially transmitting mirror is simply to reduce the amplitude of the state. Furthermore, the external coherent state is not entangled with the internal coherent state, so subsequent measurements on the external field do not physically affect the internal field if it is in a coherent state; at best they simply give more information about this internal state. Both these properties are also what might be expected for a classical field. We show in this paper that a disadvantage of using coherent states is that it obscures the important and interesting non-classical physical role that the partially transmitting mirror plays in describing the production of external light with a very pronounced intrinsic phase coherence from intra-cavity light with completely indeterminate phase. We do this by examining initially how phase-coherent external light is produced 
from an internal pure number state, which has no identifiable phase, without invoking any coherent state expansion of the internal state. This result is then extended to more general intra-cavity states. We shall see that we can attribute the external intrinsic phase coherence to the entanglement produced by the partially transmitting mirror rather than to the way in which the internal state is prepared. Indeed we find that, because of the action of the partially transmitting mirror, the phase coherence of the beam is not particularly sensitive to the intra-cavity state, provided this state has a narrow photon number distribution about a reasonably large mean.

\section{EXTERNAL FIELD PROPERTIES}

Coherence has a variety of related meanings. For example it can refer to an off-diagonal element of a density matrix or in the sense used by Glauber [22] where there are various orders of coherence. In the latter case full coherence implies that there is a Poisson distribution of photon number in one mode. In this paper we use the term phase coherence. By intrinsic phase coherence for a laser beam we mean that if we divide the beam into a sequence of packets of light of finite length and duration, as done for example in Ref. [15], then each packet will have a well-defined phase difference with every other packet. By phase we mean canonical phase as described in Appendix A. There is a close relationship between the canonical phase probability density and the off-diagonal elements of the density matrix in the number state representation [23]. If the latter all vanish, there is no identifiable phase for the field. It is important to note, however, as will be evident from this paper, that it is possible to have intrinsic phase coherence without any of the packets or the intra-cavity field having any extrinsic phase relationships, that is any identifiable phase differences with a separate phase reference system.

\section{A. First external packet}

We simplify the problem initially in order to display the essential physics by not considering the excitation of the internal cavity state. We simply let the initial state of the field in the intra-cavity mode be a pure photon number state $|N\rangle_{0}$, where $N$ is very large, and examine the phase coherence of the external light emitted from the cavity. We discuss and 
generalize this later. The cavity mode interacts with an external mode via the partially transmitting mirror. We work in the interaction picture, with the interaction Hamiltonian between the cavity mode and an external mode of the same frequency given by the energy conserving form

$$
\hat{H}_{1}=i \hbar \lambda \hat{a} \hat{b}^{\dagger}+\text { h.c. }
$$

where $\hat{a}$ and $\hat{b}$ are the photon annihilation operators for the internal and external modes respectively. If $\lambda$ is real, we have the Hamiltonian used in [23] but here we allow it to have the more general complex form $\lambda=|\lambda| \exp i \xi$.

We let the initial state be $|N\rangle_{0}|0\rangle_{1}$, that is $N$ photons in the cavity mode and a vacuum in the external mode and allow these modes to interact for a time $\delta t$ after which the interaction ceases. We discuss this in more detail later. It is not difficult to show [23] that the effect of the time displacement operator $\hat{U}_{1}=\exp \left(-i \hbar^{-1} \hat{H}_{1} \delta t\right)$ is given by

$$
\hat{U}_{1} \hat{a}^{\dagger} \hat{U}_{1}^{\dagger}=\hat{a}^{\dagger} \cos \alpha+\hat{b}^{\dagger} \exp (i \xi) \sin \alpha
$$

where $\alpha=|\lambda| \delta t$. Writing

$$
|N\rangle_{0}=(N !)^{-1 / 2}\left(\hat{a}^{\dagger}\right)^{N}|0\rangle_{0}
$$

gives

$$
\begin{aligned}
& \hat{U}_{1}|N\rangle_{0}|0\rangle_{0} \\
& =(N !)^{-1 / 2}\left(\hat{U}_{1} \hat{a}^{\dagger} \hat{U}_{1}^{\dagger}\right)^{N} \hat{U}_{1}^{N}|0\rangle_{0}|0\rangle_{1} \\
& =\left(\cos ^{N} \alpha\right) \sum_{m=0}^{N}\left(\begin{array}{l}
N \\
m
\end{array}\right)^{1 / 2} \tau^{m}|N-m\rangle_{0}|m\rangle_{1}
\end{aligned}
$$

where we have expanded $\left(\hat{U}_{1} \hat{a}^{\dagger} \hat{U}_{1}^{\dagger}\right)^{N}$ as a binomial series and $\tau=\exp (i \xi) \tan \alpha$.

\section{Photon number properties}

The probability of finding $m$ photons in the external mode and thus $N-m$ in the cavity for the entangled state (4) is

$$
P(m)=\left(\cos ^{2 N} \alpha\right)\left(\begin{array}{l}
N \\
m
\end{array}\right)|\tau|^{2 m} .
$$

The mean photon number in the external packet is thus

$$
\bar{m}=\left(\cos ^{2 N} \alpha\right) \sum_{m=1}^{N} \frac{N !|\tau|^{2 m} m}{(N-m) ! m !} .
$$


Changing the dummy variable $m$ in the summation to $n+1$ gives us eventually

$$
\bar{m}=|\tau|^{2}(N-\bar{m})
$$

Using the trigonometric relation $\sec ^{2} \alpha=1+|\tau|^{2}$ allows us to write

$$
\bar{m}=N|\tau|^{2} \cos ^{2} \alpha=N \sin ^{2} \alpha
$$

Another useful relation is

$$
\cos ^{2} \alpha=1-\bar{m} / N
$$

To find the photon number variance, we first calculate

$$
\begin{aligned}
\langle m(m-1)\rangle & =\left(\cos ^{2 N} \alpha\right) \sum_{m=2}^{N} \frac{N !|\tau|^{2 m} m(m-1)}{(N-m) ! m !} \\
& =|\tau|^{4}\langle(N-m)(N-m-1)\rangle .
\end{aligned}
$$

Although it is straightforward to find the exact result, for this paper we need only the result in the approximation that $N>\bar{m}>>1$, in which case we find from (7) that the right hand side is $\bar{m}^{2}$ to a very good approximation, yielding a number variance of

$$
\overline{m^{2}}-\bar{m}^{2} \approx \bar{m}
$$

Thus for the case $\bar{m}>>1$ the photon number distribution of the packet is quite narrow with a variance similar to that for a Poisson distribution. Indeed if we trace over the intracavity state, we find that for $N>>\bar{m}$ the reduced external density operator does have an approximate Poisson number state distribution.

\section{Phase difference properties}

The phase difference probability density for the entangled state (4) with $N-m$ and $m$

photons in the cavity and external modes respectively can be found from the formula (A7) of Appendix A. We obtain, with $n_{1}=N-m$,

$$
\mathcal{P}(\Delta)=\left.\left.\frac{\left(\cos ^{2 N} \alpha\right)}{2 \pi}\left|\sum_{m=0}^{N}\left(\begin{array}{l}
N \\
m
\end{array}\right)^{1 / 2}\right| \tau\right|^{m} \exp [i m(\Delta-\xi)]\right|^{2} .
$$

This can also be written in the more general form

$$
\mathcal{P}(\Delta)=\frac{1}{2 \pi}\left\{1+\sum_{p=1}^{N}[\langle\exp (i p \Delta)\rangle \exp (-i p \Delta)+\text { c.c. }]\right\} .
$$


where, from (A9),

$$
\begin{aligned}
& \langle\exp (i p \Delta)\rangle \\
& =\tau^{*-p}\left(\cos ^{2 N} \alpha\right) \sum_{m=p}^{N}|\tau|^{2 m}\left(\begin{array}{l}
N \\
m
\end{array}\right)^{1 / 2}\left(\begin{array}{c}
N \\
m-p
\end{array}\right)^{1 / 2} .
\end{aligned}
$$

The distribution (12) is clearly symmetric about the value $\Delta=\xi$. Thus the mean phase difference in a window from $\xi-\pi$ to $\xi+\pi$ is

$$
\langle\Delta\rangle=\xi
$$

For this paper, it is useful to find the variance of phase difference in the regime $\bar{m}>>1$, for which it is sufficient to use the approximation

$$
\left\langle\Delta^{2}\right\rangle-\langle\Delta\rangle^{2} \approx \frac{1}{2}\{1-\langle\exp [i 2(\Delta-\langle\Delta\rangle)]\rangle\}
$$

for small variances. Using (14) with $p=2$ and (15) we obtain

$$
\begin{aligned}
& \langle\exp [i 2(\Delta-\langle\Delta\rangle)]\rangle \\
& =|\tau|^{-2} \sum_{m=0}^{N} P(m)\left[\frac{m(m-1)}{(N-m+2)(N-m+1)}\right]^{1 / 2} \\
& \approx|\tau|^{-2} \sum_{m=0}^{N} P(m) \frac{m}{N}\left(1+\frac{m}{N}\right)\left(1-\frac{1}{m}\right)^{1 / 2}
\end{aligned}
$$

for $N>\bar{m} \gg>1$. For this case the number distribution is quite narrow as shown by (11), so we can approximate (17) further to become

$$
\langle\exp [i 2(\Delta-\langle\Delta\rangle)]\rangle=|\tau|^{-2} \frac{\bar{m}}{N-\bar{m}}\left[1-\frac{1}{2 \bar{m}}\right] .
$$

From (16) and (7) we obtain for the phase difference variance

$$
\left\langle\Delta^{2}\right\rangle-\langle\Delta\rangle^{2} \approx \frac{1}{4 \bar{m}}
$$

Thus there is a very well-defined phase difference between the cavity field and the first packet.

\section{B. Second external packet}

As discussed later, after the time $\delta t$ the interaction between the cavity and the first packet ceases and the packet travels away. The interaction of the cavity field with the 
second packet, initially in the vacuum state $|0\rangle_{2}$ then commences for an equal period with an interaction Hamiltonian $\hat{H}_{2}$ of similar form to (1) with $\hat{c}$, the annihilation operator for the second packet, in place of $\hat{b}$. The cavity field is no longer in the state $|N\rangle_{0}$ but is entangled with the first packet in the state (4). At the end of this second period the state of the cavity, first packet and second packet will be

$$
\begin{aligned}
& \hat{U}_{2} \hat{U}_{1}|N\rangle_{0}|0\rangle_{1}|0\rangle_{2} \\
& =\sum_{m=0}^{N} \sum_{n=0}^{N-m}\left(\begin{array}{c}
N \\
m
\end{array}\right)^{1 / 2} \tau^{m}(\cos \alpha)^{2 N-m} \\
& \quad \times\left(\begin{array}{c}
N-m \\
n
\end{array}\right)^{1 / 2} \tau^{n}|N-m-n\rangle_{0}|m\rangle_{1}|n\rangle_{2} .
\end{aligned}
$$

\section{Photon number properties}

Using (20) we find that the mean photon number in the second packet is

$$
\overline{\bar{n}}=\sum_{m=0}^{N} P(m) \bar{n}
$$

where

$$
\bar{n}=\sum_{n=0}^{N-m} P(n \mid m) n
$$

with $P(n \mid m)$, the probability that there are $n$ photons in the second packet if there are $m$ in the first packet, being given by

$$
P(n \mid m)=(\cos \alpha)^{2 N-2 m}\left(\begin{array}{c}
N-m \\
n
\end{array}\right)|\tau|^{2 n} .
$$

Using a similar derivation to that of (8), we find

$$
\bar{n}=(N-m) \sin ^{2} \alpha
$$

Then, from (9), we find for the mean number of photons left in the cavity, that is, averaged over $n$ and $m$,

$$
N-\bar{m}-\overline{\bar{n}}=(N-\bar{m}) \cos ^{2} \alpha=N \cos ^{4} \alpha .
$$

A similar calculation to that leading to (11) gives the number variance

$$
\overline{\overline{n^{2}}}-\overline{\bar{n}}^{2}=\overline{\bar{n}}
$$

Thus, as for the first packet, for large photon numbers the photon number distribution of the second packet is also quite narrow and is similar to that for a Poisson distribution. 


\section{Decay of cavity field}

The mean number of photons $\bar{m}$ in the first packet is given by (8), leaving

$$
N-\bar{m}=N \cos ^{2} \alpha
$$

as the mean cavity photon number. After the second packet is emitted the mean number left in the cavity is given by (25). It is not difficult to show that after $q$ packets the mean number left is $N \cos ^{2 q} \alpha$. We write this as $N(t)$ where $N(0)=N$. As the time taken for each packet is $\delta t$, the number remaining at time $t=q \delta t$ is

$$
N(t)=N \exp (-\Gamma t)
$$

where

$$
\Gamma=-\frac{2 \ln \cos \alpha}{\delta t} \approx \alpha^{2} / \delta t
$$

for small $\alpha$.

\section{Phase difference of second packet and cavity field}

Letting $\Delta_{21}$ be the phase difference of the cavity field and second packet, we find from (A16) that $\mathcal{P}\left(\Delta_{21}\right)$ is symmetric about $\Delta_{21}=\xi$ so the mean phase difference is $\xi$, as for the first packet. A calculation similar to that leading to (19) shows that the phase difference variance is approximately $1 / 4 \overline{\bar{n}}$. In these respects the phase properties of the second packet are similar to those of the first packet.

\section{Phase difference of first and second packets}

Now letting $\Delta_{21}$ in (A16) be the phase difference of first and second packets, we find that $\mathcal{P}$ is symmetric about $\left(\Delta_{21}\right)=0$ so the mean phase difference is zero. Then by using (16) with $\Delta_{21}$ in place of $\Delta$, we find eventually from (20) that the phase difference variance is

$$
\left\langle\Delta_{21}^{2}\right\rangle-\left\langle\Delta_{21}\right\rangle^{2} \approx \frac{1}{4 \bar{m}}+\frac{1}{4 \overline{\bar{n}}}
$$




\section{PHASE DIFFERENCE DIFFUSION}

Because the photon number operator for the first packet commutes with $\hat{U}_{2}$, the formation of the second packet as given by (20) has no effect on the photon number distribution of the first packet. This argument is not applicable, however, to the distribution of the phase difference between the first packet and the cavity. It is interesting to find what this effect actually is. We use the general expression (A16) where now $n_{0}$ refers to the second packet and $n_{1}$ and $n_{2}$ refer to the cavity and first packet fields. We first write this distribution in the form (13)

$$
\mathcal{P}(\Delta)=\frac{1}{2 \pi}\left\{1+\sum_{p=1}^{N}[\langle\exp (i p \Delta)\rangle \exp (-i p \Delta)+c . c]\right\}
$$

where, using (23),

$$
\begin{aligned}
& \langle\exp (i p \Delta)\rangle \\
& =\tau^{*-p}(\cos \alpha)^{2 N+p} \sum_{m=p}^{N}|\tau|^{2 m}\left(\begin{array}{c}
N \\
m
\end{array}\right)^{1 / 2}\left(\begin{array}{c}
N \\
m-p
\end{array}\right)^{1 / 2} \\
& \quad \times \sum_{n=0}^{N} P(n \mid m) F^{1 / 2}
\end{aligned}
$$

with

$$
F=\left(\begin{array}{c}
N-m+p \\
n
\end{array}\right)\left(\begin{array}{c}
N-m \\
n
\end{array}\right)^{-1} .
$$

In Appendix B we calculate $F$ in the large $N$ approximation and find expression (B 8 ) for $\sum P(n \mid m) F^{1 / 2}$. Substitution of (B8) into (32) gives

$$
\begin{aligned}
& \langle\exp (i p \Delta)\rangle=\tau^{*-p}\left(\cos ^{2 N} \alpha\right) \\
& \times \sum_{m=p}^{N}|\tau|^{2 m}\left(\begin{array}{c}
N \\
m
\end{array}\right)^{1 / 2}\left(\begin{array}{c}
N \\
m-p
\end{array}\right)^{1 / 2}\left[1-\frac{p^{2} \sin ^{2} \alpha}{8(N-m)}\right] .
\end{aligned}
$$

Thus we see by comparison with (14) that the change due to the formation of the second packet is quite small. The coefficients in the summation have dominant values near $m=\bar{m}$, allowing us to write the fractional change in $\langle\exp (i p \Delta)\rangle$ for $N>>\bar{m}$ as

$$
\left[1-\frac{p^{2} \sin ^{2} \alpha}{8(N-\bar{m})}\right] \text {. }
$$

The second term in the summation in (31) yields the same fractional change. Thus the change in the phase difference density $(31)$ is

$$
\delta \mathcal{P}(\Delta)=-\frac{\sin ^{2} \alpha}{16 \pi(N-\bar{m})} \sum_{p=1}^{N} p^{2}[\langle\exp (i p \Delta)\rangle \exp (-i p \Delta)
$$




$$
\begin{aligned}
& + \text { c.c. }] \\
& =\frac{\sin ^{2} \alpha}{8(N-\bar{m})} \frac{\partial^{2} \mathcal{P}(\Delta)}{\partial \Delta^{2}} .
\end{aligned}
$$

For our small $\alpha$ approximation we can replace $\sin ^{2} \alpha$ by $\alpha^{2}$ and use (29) to give

$$
\frac{\delta \mathcal{P}(\Delta)}{\delta t}=\frac{\Gamma}{8(N-\bar{m})} \frac{\partial^{2} \mathcal{P}(\Delta)}{\partial \Delta^{2}}
$$

As $N-\bar{m}$ is the mean cavity photon number at the time of the start of the second packet, expression (37) generalises to the phase difference diffusion equation

$$
\frac{\partial \mathcal{P}(\Delta)}{\partial t}=\frac{\Gamma}{8(N-\bar{m})} \frac{\partial^{2} \mathcal{P}(\Delta)}{\partial \Delta^{2}}
$$

The effect of (38) is as follows. If the phase difference probability distribution has a peak at a particular value, then the second derivative of $\mathcal{P}$ will be negative so $\mathcal{P}$ will be reduced with time, reducing the size of the peak. Similarly the value of $\mathcal{P}$ at any trough will be increased. This has the effect of changing any non-uniform phase difference distribution to a uniform distribution. When (38) is compared with the phase diffusion equation of Ref. [24], we see that the diffusion coefficient is precisely that part of the coefficient found in [24] to be attributable to the decay of the cavity field. A practical laser will, of course, also involve a gain associated with an amplification process in addition to the loss that we have considered here. This amplification will increase the phase difference noise and thus increase the diffusion.

\section{GENERAL CAVITY STATES}

Although we have considered a cavity field in a photon number state, we are not advocating interpreting the intra-cavity improper mixture with a Poisson number distribution as a pure number state of unknown photon number, even though this is as justifiable as interpreting it as a coherent state of unknown phase. Not only does considering the initial internal field as a number state make explicit the important role of the partially transmitting mirror, it can be used as a basis for examining more general fields. It is not difficult to extend the preceding calculations based on $N$ photons initially in the cavity to more general states, both superpositions and mixtures, including states in which the cavity field

is entangled with the cavity atoms. As a particular example, let us consider an initial cavity 
field in a coherent state

$$
\exp (-\bar{N} / 2) \sum_{N=0}^{\infty} \frac{\bar{N}^{N / 2} \exp (i N \zeta)}{(N !)^{1 / 2}}|N\rangle_{0}
$$

where $\zeta$ is the mean phase of the coherent state relative to some separate phase reference system. The resulting external state derived from (4) becomes

$$
\exp (-\bar{N} / 2) \sum_{N=0}^{\infty} \frac{\bar{N}^{N / 2} \exp (i N \zeta)}{(N !)^{1 / 2}} \hat{U}_{1}|N\rangle_{0}
$$

Using (4) we find eventually that (40) is a product of two coherent states: a cavity state with a mean photon number of $\bar{N} \cos ^{2} \alpha$ and a mean phase of $\zeta$, and an external state with a mean photon of $\bar{N} \sin ^{2} \alpha$ and a mean phase of $\zeta+\xi$. Thus, although both these states now have an identifiable phase relative to a separate reference system, the mean phase difference is the same as for the case when the initial cavity state is a pure number state. Further, as shown in Ref. [23], the phase variance of a coherent state $|\alpha\rangle$ with mean photon number $|\alpha|^{2}>>1$ is $\left(4|\alpha|^{2}\right)^{-1}$. Thus for our case, in which $\bar{N}>\bar{m}$, the phase difference variance is $(4 \bar{m})^{-1}$, the same as given by (19). Similarly, we find that the second packet is also in a coherent state with a mean phase of $\zeta+\xi$, so the mean phase difference between the first and second packet is zero. The variance of the phase difference between the first and second packets is again given by (30). Thus replacing the initial photon number state of the cavity field by a coherent state with the same mean photon number does not significantly change the intrinsic phase coherence of the laser light. The main effect of such a replacement is simply to give the laser light an identifiable phase relative to some external phase reference.

In general, we find that in the case where there is a reasonably well-defined cavity photon number, that is a reasonably narrow number-state distribution, we can, for example, simply replace $N$ by the mean $\bar{N}$ in (8) and (9), with the subsequent number variance given by (11) being retained. The mean phase difference is still $\xi$ as in (15) and the phase difference variance is still given approximately by (19). The essential phase coherence properties of the light are still very pronounced if the cavity photon number distribution is narrow. This is so even if the cavity field state is entangled with the cavity atoms. Thus the intrinsic phase coherence of the laser light does not depend particularly on the state of the intra-cavity field, provided it has a narrow number state distribution. It is interesting to compare this result with $[1,21]$ where we found that interference effects for independent light sources are also 
pronounced for narrow number state distributions, which perhaps is not what might have been expected intuitively, given that photon number is the complement of phase.

\section{DISCUSSION}

The state of each packet is determined mathematically by $\cos \alpha$, where $\alpha=|\lambda| \delta t$. To understand the role of the partially transmitting mirror, it is worth relating these quantities to physical properties of the laser cavity. Lee [25, 26] has examined the energy loss from a cavity of length $L$ with a fully reflecting mirror at one end and a partially transmitting mirror at the other. He found that the inverse of the decay time is

$$
\Gamma=-c \ln R /(2 L)
$$

where $R$ is the reflectivity of the partially transmitting mirror. A physical picture would associate the photons with the excitation energy of a wave in the cavity. For a cavity with two fully reflecting mirrors, the wave would be a standing wave, which can be considered as the superposition of two waves travelling in opposite directions. If one of the mirrors is suddenly removed, the component wave travelling towards that end emerges from the cavity; the component travelling in the opposite direction is reflected by the mirror at the other end and follows the first component out of the cavity, forming a travelling wave packet of length $2 L$ which has left the cavity entirely after a time $\delta t$ of $2 L / c$. In this case, for which $R$ is zero, there would be just one packet, the first packet, which would contain all $N$ photons. If, instead of removing the mirror, we replace it by a partially transmitting mirror, that is for which $R \neq 0$, then as the first packet travels through this mirror, some of it is reflected back into the cavity to form the second packet, which starts to pass through the partially transmitting mirror after a time interval $\delta t$, that is, immediately after the first packet has fully emerged. In this case the first packet will contain only an average of $\bar{m}=(1-R) N$ photons with $N-\bar{m}$ being reflected. The subsequent reflection from the second packet creates the third packet and so on. In a practical laser, of course, the second packet will be amplified as it travels double the length of the cavity before emerging. Comparing the above expression for $\bar{m}$ with (8) allows us to identify the reflectivity $R$ with $\cos ^{2} \alpha$. Then using $\delta t=2 L / c$ we see that (29) and Lee's expression (41) are fully consistent. As each packet emerges, the partially transmitting mirror entangles it with the intra-cavity field in 
such a way as to produce a well-defined phase difference between the intra-cavity field and the packet, even though both of these fields have uniform phase distributions relative to any separate phase reference system. This leads to a well-defined phase difference between different packets of the beam. In the case considered in this paper, the phase difference between a particular packet and the intra-cavity field becomes more diffuse as the intracavity field changes with the emission of subsequent packets. In a practical laser, of course, there would also amplification to balance the loss and this would result in more phase difference diffusion [27].

\section{CONCLUSION}

We have studied in this paper the light emitted from a laser cavity driven by a field in the intra-cavity mode initially in a photon number state. This state was chosen as an extreme example of a state with a uniform phase distribution relative to any separate phase reference system and which cannot be interpreted as a state of definite but unknown phase. In this way we have shown explicitly the role played by the partially transmitting mirror in creating a phase-coherent external beam. The phase coherence arises because the partially transmitting mirror entangles each packet of the beam with the intra-cavity field, creating a well-defined phase difference between them, even though neither the intra-cavity field nor the external field have an identifiable phase, that is, they have, and maintain, a uniform phase probability distribution relative to any separate phase reference. We might refer to this as "phase difference without phase". The calculations are easily generalized to other states, including mixed states and states in which the intra-cavity field is the entangled with the gain medium. The results for the phase coherence remain the same to a good approximation

for any intra-cavity field, provided the number state distribution of this field is reasonably narrow about a mean number much greater than unity. This approach can be contrasted with mathematical approaches involving expressing the intra-cavity field in terms of coherent states. Because the effect of the partially transmitting mirror on a coherent state is mainly just to diminish its amplitude without entangling it with the external state, the important role of the partially transmitting mirror is somewhat obscured by the latter approaches.

There is a parallel between the effect investigated in this paper and interference of light from two separate cavities mentioned in the Introduction, which also led to the assumption 
that the internal cavity field must be in a coherent state. Both intrinsic phase coherence and the interference that arise when the intra-cavity states are number states are quantum effects relying on entanglement. In the latter case, entanglement allows the measurement itself to create the phase difference between the states of the two cavities that is being measured. This phase difference develops without either of the cavity fields having or acquiring a welldefined phase relative to any separate reference system. In the case studied in this paper entanglement allows the internal field and different parts of the external field of one cavity to have well-defined phase differences without any having a well-defined phase relative to any separate reference system. This, of course only applies within the phase difference diffusion time, which also follows straightforwardly from the approach adopted in this paper. We note that the diffusion equation for phase difference is similar to that usually applied to phase. The phase difference diffusion equation of this paper describes how the initial sharply peaked phase difference distribution between a packet and the intra-cavity field that is created when the packet is formed diffuses into a uniform distribution. The usual phase diffusion equation, on the other hand, describes how a sharply peaked phase distribution of the intra-cavity field would diffuse if one were created.

In this paper it has been shown that it is certainly not necessary for the intra-cavity laser field to be in a coherent state for the emitted light to have intrinsic phase coherence, although it is sufficient. Indeed the phase coherence is not particularly dependent on the actual intra-cavity state. Of course, this by itself does not mean that the internal and emitted fields cannot be in coherent states. It is well known that measurements that do not involve the atoms of the gain medium cannot distinguish among different partitions of the reduced internal density operator [14]. Determining whether or not the cavity field is in a coherent state would require either measurements involving the atoms of the gain medium [1] or detailed consideration of the preparation procedure [6]. We do not wish enter this debate here. The purpose of this paper has been to examine the normally overlooked role of the partially transmitting mirror in creating phase-coherent laser light from almost any intra-cavity state, even a photon number state or an incoherently excited state entangled with the gain medium. 


\section{APPENDIX A: EXPRESSIONS FOR PHASE DIFFERENCE}

The phase observable used in this paper is sometimes called the canonical phase [28]. It can be defined as the complement of the photon number [29] or in terms of a probability operator measure [30]. It can also be defined operationally as the quantity that is shifted by a phase shifter[28, 31]. All these approaches are consistent and yield the same phase probability distributions.

The phase wavefunction for a number state $|n\rangle$ is given by [31]

$$
\psi(\theta)=(2 \pi)^{-1 / 2} \exp (-i n \theta)
$$

which is normalized over any $2 \pi$ range. From this, the phase probability density for a state

$$
\left|f_{1}\right\rangle=\sum_{n} c_{n}|n\rangle
$$

is given by

$$
\mathcal{P}(\theta)=\frac{1}{2 \pi}\left|\sum_{n} c_{n} \exp (-i n \theta)\right|^{2} .
$$

This quantity can be measured by projection synthesis and other methods [32]. It is not difficult to show from (A3) that for a field with a diagonal density matrix in the number state representation the phase probability distribution is uniform, that is, there is no identifiable phase.

For a two-field state

$$
\left|f_{2}\right\rangle=\sum_{n_{1}, n_{2}} c_{n_{1}, n_{2}}\left|n_{1}\right\rangle\left|n_{2}\right\rangle
$$

the joint phase probability density is

$$
\mathcal{P}\left(\theta_{1}, \theta_{2}\right)=\frac{1}{4 \pi^{2}}\left|\sum_{n_{1}, n_{2}} c_{n_{1} n_{2}} \exp \left[-i\left(n_{1} \theta_{1}+n_{2} \theta_{2}\right)\right]\right|^{2} .
$$

By putting $\Delta=\theta_{2}-\theta_{1}$ and integrating over all values of $\theta_{1}$ in a $2 \pi$ range we obtain the phase difference probability density [31]

$$
\begin{aligned}
\mathcal{P}(\Delta)= & \frac{1}{2 \pi} \sum_{n_{1}, n_{2}} \sum_{m_{1}}\left\{c_{n_{1} n_{2}}^{*} c_{m_{1}\left(n_{1}-m_{1}+n_{2}\right)}\right. \\
& \left.\times \exp \left[i\left(m_{1}-n_{1}\right) \Delta\right]\right\} .
\end{aligned}
$$

For the special case where $n_{1}+n_{2}=N$, a constant, this simplifies to become

$$
\mathcal{P}(\Delta)=\frac{1}{2 \pi}\left|\sum_{n_{1}} c_{n_{1}\left(N-n_{1}\right)} \exp \left(-i n_{1} \Delta\right)\right|^{2},
$$


which has the same form as (A3). Thus in this special case the phase difference distribution is the same as the phase distribution of a single pure state with appropriate number state coefficients. Expression (A7) can also be written in the more general form

$$
\mathcal{P}(\Delta)=\frac{1}{2 \pi}\left\{1+\sum_{p=1}^{N}[\langle\exp (i p \Delta)\rangle \exp (-i p \Delta)+\text { c.c. }]\right\}
$$

with

$$
\langle\exp (i p \Delta)\rangle=\sum_{n_{1}=0}^{N-p} c_{n_{1}\left(N-n_{1}\right)} c_{\left(n_{1}+p\right)\left(N-n_{1}-p\right)}^{*} .
$$

For the three-field state

$$
\left.\left|f_{3}\right\rangle=\sum_{n_{0}} \sum_{n_{1}} \sum_{n_{2}} c_{n_{0} n_{1} n_{2}}\left|n_{0}\right\rangle\left|n_{1\rangle}\right| n_{2}\right\rangle
$$

the joint phase probability density is

$$
\begin{aligned}
& \mathcal{P}\left(\theta_{1}, \theta_{2}, \theta_{3}\right)=\frac{1}{8 \pi^{3}} \\
& \times\left|\sum_{n_{0} n_{1} n_{2}} c_{n_{0} n_{1} n_{2}} \exp \left[-i\left(n_{0} \theta+n_{1} \theta_{1}+n_{2} \theta_{2}\right)\right]\right|^{2} .
\end{aligned}
$$

To find the probability for two of the fields to have a phase difference $\Delta_{21}=\theta_{2}-\theta_{1}$, we put $\theta_{2}=\Delta_{21}+\theta_{1}$ and integrate over all values of $\theta_{1}$ and $\theta_{2}$ in a $2 \pi$ range:

$$
\begin{aligned}
\mathcal{P}\left(\Delta_{21}\right) & =\frac{1}{8 \pi^{3}} \int_{2 \pi} d \theta_{0} \int_{2 \pi} d \theta_{1} \sum_{n_{0} n_{1} n_{2}} \sum_{m_{0} m_{1} m_{2}}\left\{c_{n_{0} n_{1} n_{2}}\right. \\
& \times c_{m_{0} m_{1} m_{2}}^{*} \exp \left\{-i\left[\left(n_{0}-m_{0}\right) \theta_{0}\right.\right. \\
& \left.\left.+\left(n_{1}-m_{1}\right) \theta_{1}+\left(n_{2}-m_{2}\right)\left(\Delta_{21}+\theta_{1}\right)\right]\right\}
\end{aligned}
$$

where the integrals can be over any $2 \pi$ range. Using

$$
\int_{2 \pi} \exp \left[-i\left(n_{0}-m_{0}\right) \theta_{0}\right] d \theta_{0}=2 \pi \delta_{n_{0} m_{0}}
$$

and

$$
\int_{2 \pi} \exp \left[-i\left(n_{1}-m_{1}+n_{2}-m_{2}\right) \theta_{1}\right] d \theta_{1}=2 \pi \delta_{\left(n_{1}-m_{1}\right)\left(m_{2}-n_{2}\right)}
$$

gives

$$
\begin{aligned}
\mathcal{P}\left(\Delta_{21}\right)= & \frac{1}{2 \pi} \sum_{n_{0}, n_{1}} \sum_{n_{2}, m_{1}}\left\{c_{n_{0} n_{1} n_{2}} c_{n_{0} m_{1}\left(n_{1}-m_{1}+n_{2}\right)}^{*}\right. \\
& \left.\times \exp \left[i\left(n_{1}-m_{1}\right) \Delta_{21}\right]\right\} .
\end{aligned}
$$

For the special case where $n_{0}+n_{1}+n_{2}=N$, a constant, this simplifies to become

$$
\mathcal{P}\left(\Delta_{21}\right)=\frac{1}{2 \pi} \sum_{n_{0}}\left|\sum_{n_{1}} c_{n_{0} n_{1}\left(N-n_{0}-n_{1}\right)} \exp \left(i n_{1} \Delta_{21}\right)\right|^{2},
$$




\section{APPENDIX B: CALCULATION FOR SECTION III}

We can write $F$ in $(33)$ as

$$
F=\frac{(N-m)^{p} \prod_{r=1}^{p}[1+r /(N-m)]}{(N-m-n)^{p} \prod_{r=1}^{p}[1+r /(N-m-n)]} .
$$

For the case we are considering, that is where $N$ is much larger than the dominant values of $m$ and $n$ in (32), we can use the expansion in powers of $n$

$$
F=\left(\frac{N-m}{N-m-n}\right)^{p}\left[1-\frac{p(p+1) n}{2(N-m)^{2}}+\ldots\right]
$$

with

$$
\left(\frac{N-m}{N-m-n}\right)^{p / 2}=1+\frac{p n}{2(N-m)}+\frac{p(p+2) n^{2}}{8(N-m)^{2}}+\ldots
$$

to obtain

$$
\begin{aligned}
\sum_{n=0}^{N} P(n \mid m) F^{1 / 2}= & 1-\frac{p(p+1) \bar{n}}{4(N-m)^{2}}+\frac{p \bar{n}}{2(N-m)} \\
& +\frac{p(p+2) \overline{n^{2}}}{8(N-m)^{2}}+\ldots
\end{aligned}
$$

A calculation similar to that leading to (11) with $N-m$ in place of $N$ yields, for $N>>\bar{n}$

$$
\overline{n^{2}} \approx \bar{n}^{2}+\bar{n}+f(m)
$$

where $f(m)$ is negligible for $m<<N$, which are the dominant values of $m$ in (32). Substituting for $\overline{n^{2}}$ from (B5), neglecting the term $f(m)$, we find in the large $n$ approximation that (B4) becomes

$$
\begin{aligned}
& \sum_{n=0}^{N} P(n \mid m) F^{1 / 2} \\
& \approx 1+\frac{p \bar{n}}{2(N-m)}+\frac{p(p+2) \bar{n}^{2}}{8(N-m)^{2}} \\
& \quad+\frac{p(p+2) \bar{n}}{8(N-m)^{2}}-\frac{p(p+1) \bar{n}}{4(N-m)^{2}} \\
& \approx\left[1-\frac{\bar{n}}{N-m}\right]^{-p / 2}\left[1-\frac{p^{2} \bar{n}}{8(N-m)^{2}}\right] \\
& \approx(\cos \alpha)^{-p}\left[1-\frac{p^{2} \sin ^{2} \alpha}{8(N-m)}\right]
\end{aligned}
$$

where we have used (24). 


\section{ACKNOWLEDGMENTS}

I thank Joan Vaccaro for discussions and Daniel Pegg for his support. This research was funded in part by the Australian Research Council.

[1] D. T. Pegg, Phys. Rev. A74, 063812 (2006).

[2] R. L. Pfleegor and L. Mandel, Phys. Lett. A 24, 766 (1967).

[3] R. L. Pfleegor and L. Mandel, Phys. Rev. 159, 1084 (1967).

[4] S. J. Kuo, D. T. Smithey, and M. G. Raymer, Phys. Rev. A 43, 4083 (1991).

[5] P. Hariharan, N. Brown, and B. C. Sanders, J. Mod. Opt. 40, 113 (1993).

[6] K. Mølmer, Phys. Rev. A 55, 3195 (1997); K. Mølmer, J. Mod. Opt. 44, 1937 (1997).

[7] H. Cable, P. L. Knight, and T. Rudolph, Phys. Rev. A 71, 042107 (2005).

[8] R. Ghosh and L. Mandel, Phys. Rev. Lett. 59, 1903 (1987).

[9] D. T. Pegg, Aust. J. Phys. 46, 77 (1993).

[10] J. Javanainen and S. M. Yoo, Phys. Rev. Lett. 76, 161 (1996).

[11] P. Horak and S. M. Barnett, J. Phys. B 32, 3421 (1999).

[12] J. Gea-Banacloche, Phys. Rev. A 58, 4244 (1998).

[13] K. Mølmer, Phys. Rev. A 58, 4247 (1998).

[14] T. Rudolph and B. C. Sanders, Phys. Rev. Lett. 87, 077903 (2001).

[15] S. J. van Enk and C. A. Fuchs, Phys. Rev. Lett. 88, 027902 (2001).

[16] B. C. Sanders, S. D. Bartlett, T. Rudolph, and P. L. Knight, Phys. Rev. A, 68, 042329 (2003).

[17] H. M. Wiseman, J. Mod. Opt. 50, 1797 (2003): H. M. Wiseman. J. Opt. B 6 S849 (2004).

[18] M. Fujii, Phys. Rev. A68 050302(R) (2003).

[19] R. W. Spekkens and J. E. Sipe, in Coherence and Quantum Optics VIII, eds. N. P. Bigelow, J. H. Eberly, C. R. Stroud, Jr., and I. A. Walmsley (Kluwer Academic, New York, 2003) p. 465.

[20] S. D. Bartlett, T. Rudolph, and R. W. Spekkens, Int. J. Quant. Information 4, 17 (2006); arXiv:quant-ph/0507214v2 (2005).

[21] D. T. Pegg and J. Jeffers, J. Mod. Opt. 52, 1835 (2005).

[22] R. J. Glauber, in Quantum Optics, eds. S. M. Kay and A. Maitland (Academic Press, London, 
1970) p. 53.

[23] S. M. Barnett and P. M. Radmore, Methods in Theoretical Quantum Optics (Oxford University Press, Oxford, 1997).

[24] S. M. Barnett, S. Stenholm, and D. T. Pegg, Opt. Commun. 73, 314 (1989).

[25] C. T. Lee, Opt. Commun. 27, 277 (1978).

[26] C. T. Lee, Phys. Rev. A 48, 2285 (1993).

[27] J. A. Vaccaro and D. T. Pegg, Phys. Rev. A 49, 4985 (1994).

[28] U. Leonhardt, J. A. Vaccaro, B. Böhmer, and H. Paul, Phys. Rev. A 51, 84 (1995).

[29] D. T. Pegg and S. M. Barnett, Europhys. Lett. 6, 483 (1988); S. M. Barnett and D. T. Pegg, J. Mod. Opt. 36, 7 (1989); D. T. Pegg and S. M. Barnett, Phys. Rev. A 39, 1665 (1989).

[30] J. H. Shapiro and S. R. Shepard, Phys. Rev. A 43, 3795 (1991); J. A. Vaccaro and D. T. Pegg, Physica Scripta T48, 22 (1993).

[31] D. T. Pegg and S. M. Barnett, J. Mod. Opt. 44, 225 (1997).

[32] S. M. Barnett and D. T. Pegg, Phys. Rev. Lett. 76, 4148 (1996); K. L. Pregnell and D. T. Pegg, J. Mod. Opt. 49, 1135 (2002); K. L. Pregnell and D. T. Pegg, Phys. Rev. A 67, 063814 $(2003)$. 